\title{
MACHISMO Y RELIGIOSIDAD: ELEMENTOS CULTURALES QUE ENMARCAN LA LGBTIFOBIA EN (LA UNIVERSIDAD DE) COSTA RICA
}

\author{
Male chauvinism and religiousness: \\ cultural elements that enlarge LGTBIphobia in (the University of) Costa Rica
}

\author{
María del Carmen Acuña Rodríguez \\ Universidad de Costa Rica, Costa Rica \\ mariadelcarmen.acuna@ucr.ac.cr
}

Recibido: 15-02-2017

Aprobado: 04-05-2017

\begin{abstract}
María del Carmen Acuña Rodríguez es Psicóloga, Educadora, Evaluadora; Activista feminista y LGBTI. Ha laborado para la Universidad de Costa Rica en diferentes instancias. Ha sido asesora académica para docentes y carreras universitarias (en procesos curriculares y de evaluación), profesora (en cursos de sexualidad y educación sexual), e investigadora (en temas de educación superior, género, sexualidad y diversidad sexual). Actualmente labora en el Centro de Evaluación Académica de la UCR y como consultora para universidades.
\end{abstract}

\section{RESUMEN}

El artículo aborda dos elementos culturales de la identidad nacional en Costa Rica, que se vinculan al tema de la disidencia sexual y de género como productores de prejuicios hacia personas LGBTI, a partir de lo que se estudió en un contexto específico: la Universidad de Costa Rica (UCR). Dichos elementos son el machismo y la religiosidad; se articulan como componentes centrales de la cultura costarricense que inciden directamente en prácticas de discriminación y violencia hacia dicha población. Las reflexiones que se presentan son parte de los resultados de la investigación titulada "En la ruta del arco iris: vivencias de personas LGBTI de la Universidad de Costa Rica dentro del mundo universitario". En ella participaron estudiantes, docentes y administrativxs de la institución, cuya característica es tener identidades sexuales y de género fuera de la norma heterosexual. La UCR funciona como micro-mundo inserto en la vida y la cultura del país, que interactúa e incide en ellas. Dentro del espacio universitario (en el nivel micro) se reproducen prácticas culturales que ocurren en lo macro. La Universidad debería reflexionar acerca de ellas y promover cambios, especialmente cuando se trata de cuestiones que excluyen y vulnerabilizan más a grupos marginados.

Palabras clave: diversidad sexual; Universidad de Costa Rica; machismo; religión

\section{ABSTRACT}

This paper addresses two cultural elements, about the Costa Rican national identity, related to sexual-gender dissidence as producers of stereotypes towards LGBTI people, based on what was studied in a specific context: the Universidad de Costa Rica (UCR). Those elements are machismo (male chauvinism) and religiousness; they are assembled as important components of the Costa Rican culture which directly influences discrimination and violence towards this population. The reflections presented here are part of the results of a research entitled "On the rainbow's path: life experiences of LGBTI people from the Universidad de Costa Rica inside the university world". It contains the participation of the institution's students, teachers and administrative workers, who have 
sexual and gender identities out of the heterosexual norm. The UCR functions as a microworld inside the country's life and culture, but also interacts and influences them. Inside the university space (in a micro level) there is a reproduction of cultural practices that happen in a macro level. The University should reflect this and promote changes, especially when such practices exclude and make marginalized groups more vulnerable.

Keywords: sexual diversity; Universidad de Costa Rica; male chauvinism; religión

\section{Introducción}

En este artículo analizo elementos culturales de la idiosincrasia costarricense que están vinculados a la LGBTIfobia que existe en el país. Lo que presento es parte de un trabajo de investigación mayor, titulado "En la ruta del arco iris: vivencias de personas LGBTI de la Universidad de Costa Rica dentro del mundo universitario"1. Se trata de un estudio acerca de la disidencia sexual y de género (en adelante, DSG), desde la perspectiva de lo que han vivido personas no heterosexuales en un contexto específico, la Universidad de Costa Rica (UCR), o mejor dicho, la sede central Rodrigo Facio.

Fue una investigación cualitativa, descriptiva, con estrategia metodológica de estudio de caso, cuya intención (objetivo general) fue comprender las vivencias que, en el contexto de la UCR, han tenido personas que se autoidentifican con identidades no heteronormativas, respecto a experiencias en torno a su sexualidad disidente vividas dentro del espacio universitario; trabajé con las tres poblaciones que pertenecen a la comunidad universitaria: estudiantes, docentes y administrativxs. La herramienta para trabajar con las personas fueron diálogos, fundamentados en las propuestas de la Interculturalidad (la Filosofía Intercultural); para analizar la información obtenida, desarrollé un análisis de discurso bajo la perspectiva foucaultiana. Por tanto, en la redacción del texto, incluyo frases (citas) de las personas con quienes conversé, pues retomar sus propias palabras fue una decisión investigativa y política. En lo que presento aquí, también recurro a ello; de tal manera, junto con mis reflexiones y las citas de autorxs, se encontrarán también palabras de las personas participantes de la investigación.

En el estudio se visibilizaron múltiples formas de discriminación hacia la población LGBTI; también iniciativas de reconocimiento y afirmación que

\footnotetext{
1 Dicho trabajo fue desarrollado en los años 2015-2016; corresponde a la investigación que constituye mi tesis de maestría, en el Programa de Pós-graduação em Educação, de la Universidade Federal do Rio Grande do Sul (Brasil), bajo orientación de la profesora Dra. Magali Mendes de Menezes, en la línea de investigación Universidade: teoria e prática.
} 
las mismas personas desarrollan para intentar convertir a la UCR en un espacio que realmente sea inclusivo y libre de discriminación. Tanto la discriminación y la violencia (bajo la forma de LGBTIfobia), como las experiencias positivas, están atravesadas por elementos de la cultura del país que sirven de antesala para todas las situaciones. Es por ello que en este artículo abordo prácticas culturales relacionadas con las vivencias de personas LGBTI, como elementos que en sí mismos son importantes de analizar.

Todas las cuestiones que describo no son exclusivas a la universidad, pero se reproducen en ella; bien lo expresó un profesor: "lo que pasa en la $U$ pasa en la parte externa" (Ricardo). Esto debido a que la UCR funciona como un micro-mundo dentro del país, donde inclusive se hace referencia a ella con la metáfora de una burbuja: la universidad es una burbuja, en el sentido que dentro de la institución se vive un mundo propio, delimitado en sí mismo y a veces aislado del resto de la sociedad ${ }^{2}$. No en vano se habla del 'mundo universitario', como micro-universo a lo interno de un contexto mayor; por eso se habla de "la burbuja UCR". Y es que la UCR opera como un Estado en pequeño: tiene su propio territorio (un campus central y varias sedes regionales), símbolos identitarios, gobierno, legislación interna y formas de funcionamiento. Estamos entonces ante un micro-mundo, que interactúa e incide en el contexto mayor que lo acoge. Un estudiante lo describe así: "la $U$ es una burbuja, pero sigue estando infiltrada en la realidad; digamos, es como una micro-realidad" (Felipe, estudiante). Son dos niveles de realidad que se tocan y afectan mutuamente. Además, la cultura de un país está enmarcada en un contexto supranacional, donde se han construido discursos cerrados y prácticas de exclusión y discriminación (Margulis, 1997).

De los elementos culturales imperantes en la sociedad costarricense, que tienen una fuerte injerencia en la vida de las personas habitantes del territorio, existen dos que se relacionan directamente con la sexualidad y, por ende, con la disidencia sexual y de género: el machismo y la religiosidad. Cada uno individualmente y la conjunción de ambos es la base principal de todas las prácticas que se viven en torno a la sexualidad (y atañen a la DSG); "la vasta presencia del catolicismo (instituciones y creencias) y el valor cultural del machismo perpetúan los valores heteronormativos ${ }^{3 \prime}$ (Abelove, 2015, p. 18). Inclusive, ambos elementos

\footnotetext{
2 Esto tiene aspectos positivos y negativos, pero es una discusión más profunda y totalizadora, que queda fuera del alcance de este trabajo.

3 Traducido por mí del texto original en inglés.
} 
aparecen reiteradamente en los discursos de las personas. A continuación me refiero a ellos a partir de lo que se vive en el contexto de la UCR.

\section{El machismo versión "tiquicia"}

Existe una cultura nacional traducida en una identidad homogénea, la cual fue construida a finales del siglo XIX por parte de los grupos hegemónicos que gobernaban el país (la oligarquía cafetalera y los políticos liberales); utilizaron la educación pública para ello y tuvieron una importante contribución de la Iglesia Católica (Molina, 2015; Rosabal-Coto, 2016). La imagen nacional es ciertamente muy fuerte, me refiero a la idea del "ser costarricense", la "idiosincracia tica" y el "pura vida"4. En las entrañas de las 'costumbres y tradiciones' ticas, está el machismo, arraigado con muchísima fuerza. Así lo dijo otro docente: "yo no puedo dejar de ver machismo y patriarcado sin la cultura tica, es uno de los pilares que ella tiene; y eso es básicamente lo que se ve en las relaciones sociales en todos estos ambientes" (Enrique). En todos los diálogos, el tema de fondo que más surgió fue el machismo, haciendo también un vínculo entre este y la cultura tica.

El machismo es el 'culto' a la masculinidad, donde los hombres son/deben ser los dominantes, lo cual afecta los roles de hombres y mujeres (Abelove, 2015). Debe entenderse en el marco de la cultura patriarcal, la cual "se basa en relaciones de desiguales, tanto de poder como de privilegios" (Salas y Campos, 2002, p. 204). En Costa Rica, muchas de las prácticas culturales tienen en su base esa cultura machista-patriarcal:

\footnotetext{
Manifestaciones de la subcultura tica, pero que tienen que ver con el ser varón. Que si alguien se equivoca, todo mundo le silba. En mis clases, yo siempre le digo a mis estudiantes que van a encontrar respeto y apertura, pero no van a encontrar permisividad, aquí nadie silba, nadie chotea, esas cosas de la subcultura tica (Enrique, docente).
}

La identidad nacional retrata al país como "una feliz arcadia tropical, blanca, pacífica, rural, igualitaria y con las funciones de género claramente definidas" (Molina, 2015, p.111). Enfatizo en la última idea: los roles de género bien establecidos. Una de las maneras más fáciles de verlo es en los bailes $y$, especialmente, los folklóricos: las parejas se forman por un hombre y una mujer, donde quien tiene que 'llevar' (guiar en el baile) siempre es el hombre. Una estudiante lo explica: la mujer aprende a usar

\footnotetext{
${ }^{4}$ Por motivos de espacio, la discusión sobre la identidad nacional queda fuera del alcance de este artículo, pero también es importante realizarla.
} 
la enagua, el hombre aprende a usar el sombrero y los pañuelos; "yo quería ser el que llevaba el sombrero y no me dejaron [...] tuve que usar enagua [...] los profes te ven y te dicen si usted es una mujer, tiene que usar enagua" (Leonor).

Otra manifestación es la censura o impedimento hacia los hombres de mostrar sus sentimientos, al punto que -quienes siguen esos patrones hegemónicos- lo hacen cuando han ingerido alguna sustancia, fundamentalmente licor. Diana, una funcionaria administrativa, afirma: "es triste porque usted ve a los de ingeniería todos ebrios y es 'mae, te amo y adoro' o sea, tienen que estar alcoholizados para tener permiso de abrazarse" (Diana).

Es muy evidente que ese machismo se encuentra profundamente arraigado en el país: "la cultura machista está muy bien sostenida y todos ayudamos a sostenerla" (Enrique, docente). Inclusive, está presente en quienes de alguna manera rompen con los mandatos de ese sistema hetero-patriarcal, como las mismas personas LGBTI en muchos casos; un estudiante lo manifestó adecuadamente: "hay gays super machistas y lesbianas super machistas" (Felipe). Ello coincide con lo que Enrique profesor- observa: "puedo ver el machismo en las interacciones, de cómo todos participamos de esas interacciones, nos sintamos discriminados o no, siempre participamos de esas interacciones".

Ahora, para entender el machismo, debemos hablar de género. El género son todos los aspectos psicológicos, sociales y culturales que, en un contexto determinado, son establecidos para las personas de acuerdo con su sexo biológico (Campos y Salas, 2002). El concepto engloba "todas las formas de construcción social, cultural y lingüística implicadas con los procesos que diferencian mujeres de hombres, incluyendo aquellos procesos que producen sus cuerpos, distinguiéndolos y separándolos como cuerpos dotados de sexo, género y sexualidad" ${ }^{5 "}$ (Meyer, 2013, p.18). En ese sentido, podríamos entender el género como un marco, en tanto es "algo externo que se coloca sobre el sujeto para darle un sentido ya forjado, un marco de legibilidad social y sexual que encuadra a las personas" (García, 2015, p. 44). Ese encuadramiento se establece en dos géneros dicotómicos, es un encasillamiento en dos polos.

Una aclaración básica: sexo se refiere a lo biológico, mientras que género a lo socio-histórico-cultural. Somos la única especie cuyos machos y hembras son llamados 'hombres' y 'mujeres', y para quienes son

\footnotetext{
5 Traducción mía del texto original en portugués.
} 
establecidos tantos mandatos. Todas las manifestaciones del género son construidas por las sociedades, en un tiempo y espacio específicos, legitimando únicamente ciertas expresiones y excluyendo otras, con lo cual se imponen modelos. Se trata de los scripts ${ }^{6}$ de género y de los marcos de género (García, 2015). "Veo patriarcado y, cuando se trata de colegas varones, veo el machismo en cómo se define qué es ser varón" (Enrique, docente). Y tiene aplicación en todos los ámbitos de la vida.

Un ejemplo: una estudiante trans habla de su experiencia en la Actividad Deportiva7: "separan hombres y mujeres, se matricula por separado" (Zoé). Subyace la idea de que los dos géneros dicotómicos (los cuales ordenan las relaciones humanas) se constituyen como elementos "complementarios y excluyentes, asignados al nacer y construidos en un único sentido acorde con dicha asignación" (García, 2015, p. 44). Se configuran como atributos binarios $y$, por ello, excluyentes; es decir, lo que está presente en un género, es opuesto en el otro. Siguiendo con la experiencia que compartió Zoé, ella cuenta que la colocaron en el grupo de hombres y que, cuando le preguntó a la profesora por qué se realiza esa división en grupos de mujeres y de hombres, la respuesta de la docente fue "es que las mujeres son más débiles".

Para evitar que las personas no trasciendan (se salgan) de los marcos de género, son aplicados múltiples mecanismos de opresión. Interesante es que esto demuestra cuanto la supuesta heterosexualidad es una construcción cultural, pues "si fuera natural la cultura no necesitaría de su insistencia reiterada, llegando incluso, a veces, a límites que rozan la obsesión" (García, 2015, p. 50). Irónicamente, uno de los principales discursos contra las disidencias sexo-genéricas es que son antinaturales. Discurso que es, en gran medida, religioso.

\section{La (no) laicicidad de la UCR}

La religión es una práctica cultural que tiene muchísima incidencia en la universidad, pese a que no debería, por ser esta un espacio laico y autónomo. En ese sentido, la universidad "se mueve a la misma línea que

\footnotetext{
6 Término acuñado recientemente por la profesora e investigadora Dra. Jane Felipe de Souza, de la Universidad Federal do Rio Grande do Sul (Brasil). Tuve conocimiento de ello gracias a la colaboración académica que tuve con dicha autora durante mi posgrado en esa universidad; sin embargo, el trabajo al respecto está en fase de elaboración.

${ }^{7}$ La Actividad Deportiva es un curso de libre elección que todx estudiante de la UCR debe realizar, como parte de los cursos de formación humanística. Todos los cursos duran un semestre y están a cargo de la Escuela de Educación Física y Deportes.
} 
el país" (Lepolt, docente). Es tanto el poder que la religión tiene dentro de la institución, particularmente la católica (y dentro de ella, el Opus Dei), que uno de los profesores la llama "el Estado Pontificio de la UCR". A veces, pareciera que realmente la universidad funciona como una sucursal del Vaticano.

Como sabemos, las creencias religiosas tienen una gran influencia en las percepciones respecto al matrimonio y la familia (Abelove, 2015), lo cual incide también en las posturas sobre la población LGBTI. Una muestra de ello: Mar, unx profesorx expresa que ha recibido rechazo por parte de sus colegas y de quien fue la directora de la escuela donde trabaja, incluso perjudicándolx laboralmente, pues cuando salió del closet, esa directora le comenzó a reducir su carga académica (la jornada de trabajo) al mínimo. Se torna evidente que "la religión y la moral judeocristiana incentivan el prejuicio contra todas las formas de placer no vinculadas a la reproducción" (Cornejo, 2012, p.87). Otro ejemplo lo relata una estudiante, Leonor: en su escuela, una profesora que lee la biblia dentro de la universidad, es quien impidió el desarrollo de una iniciativa que ella (la estudiante) tuvo, de brindar talleres de sexualidad para sus compañerxs.

Varias personas -docentes y estudiantes- resaltan la presencia tan fuerte que tiene la religión y los grupos religiosos dentro de la institución universitaria, que si fuera privada y confesional sería esperable, pero es una universidad pública y laica, que se proclama humanista y respetuosa de los derechos humanos. Destaca la injerencia del Opus Dei, un sector ultraconservador de la Iglesia Católica: "el Opus Dei está tomando fuertemente la UCR, los maes tienen un plan, [...] han logrado que algunas personas se vayan a hacer reservas de plazas y después volverán y van a tener voto" (Mar, docente).

Para nadie es un secreto que, en muchas unidades académicas, la máxima figura de autoridad (sea en escuelas, facultades o sedes) es una persona con fuertes costumbres religiosas, incluso vinculadas a dicho grupo católico. Además de lo compartido en los diálogos durante la investigación, también he sido testigo de situaciones en esa línea. Por ejemplo: llegar a la oficina de la directora de una escuela, que tenía en la pared un enorme retrato del papa Juan Pablo Il; también, presenciar en un conversatorio con una profesora invitada internacional, que una autoridad universitaria se presentó diciendo 'por la gracia de Dios, yo soy la directora de la escuela...'. La lista de situaciones podría ser interminable. Y todo esto es inadmisible en una institución de educación superior estatal. 
Necesitamos entender cómo se constituyen esos discursos y cómo se justifica que existan dentro de una institución académica (en principio) laica y autónoma.

Así lo plantea, de manera clara y vehemente, una profesora:

me parece injusto que yo porque estoy en una Escuela que es activamente pro derechos humanos, como lo es Lenguas Modernas, me preocupa que en otras escuelas -y más aún, en otras sedes como en Limón, donde hay posters de Jesús en las aulas-, eso está mal a otro nivel. $O$ sea, un estudiante gay en la sede de Limón ¿qué hace?, ¿cómo se permite que en la UCR haya pósters de Jesús en los baños, en las clases? Eso es inmoral. (Adriana, docente).

Efectivamente, "eso es una barbaridad" y "está mal a otro nivel", como manifiesta Adriana. En esa misma línea, en un grupo de diálogo distinto, reflexiona un estudiante: "¿cómo tenés un sistema de pesos y contrapesos que te permita que una persona así quede electa en un cargo público, sin que su moral sea lo que aplique como política?" (Felipe). Dejo ahí la pregunta.

Tomando las palabras de otra alumna "obviamente cada quien es libre de creer lo que quiera, y de profesar lo que quiera profesar" (Ada, estudiante). Sin embargo, cada quien debería hacerlo fuera del espacio universitario, pues es un asunto de su vida personal que no debería llevar a un espacio académico-laboral, ni mucho menos obligar a otras personas a seguir sus creencias (pues eso es lo que sucede en la mayoría de los casos). Hablo que es una obligación, dado que elementos religiosos (católicos y cristianos) impregnan la vida universitaria, obligando a las personas en ella a tener un acercamiento o a entrar en ese mundo, lo quieran o no (ya lo veremos con ejemplos concretos.)

Como si el poder e influencia de las religiones católico-cristianas no fuera suficiente, los grupos religiosos de la universidad querían tener-dentro de ella- un lugar físico para desarrollar sus prácticas. En el último Congreso Universitario ${ }^{8}$, desarrollado en el año 2015, hubo una propuesta para crear un "espacio para la espiritualidad" en el campus central de la UCR. La idea era que la universidad construyese un centro donde las personas de la

\footnotetext{
${ }^{8}$ El Congreso Universitario (también llamado Congreso de la Universidad de Costa Rica) es un espacio que ocurre aproximadamente cada 10 años, donde la universidad se reúne para reflexionar en torno a sí misma. En él se plantean propuestas de creación o modificación dentro de la misma universidad, todo con el propósito de mejorar su funcionamiento y sus labores en las tres áreas sustantivas docencia, investigación y acción social. En el Congreso participan las tres poblaciones integrantes de la comunidad universitaria: docentes, administrativxs y estudiantes. Cambios importantes que han modificado significativamente el quehacer de la UCR fueron gestado en el seno de un Congreso Universitario.
} 
comunidad universitaria pudieran acudir para practicar sus ritos, el cual albergaría, supuestamente, distintas denominaciones religiosas. La propuesta' fue la siguiente:

UNIVERSIDADDE
COSTARICA
PONENCIA: UN ESPACIO PARA LA ESPIRITUALIDAD
PONENTES: Rita Porras León
ÁREA TEMÁTICA: Vida Universitaria
RESUMEN: La comunidad universitaria que da vida a la Universidad de Costa Rica conjuga
sus actividades laborales con su vida espiritual. Se propone que la Universidad edifique un
centro de espiritualidad interdenominacional que le permita los miembros de la comunidad
universitaria que asi lo deseen congregarse, reflexionar, dialogar, estudiar críticamente y
practicar los ritos de su fe. Este espacio estaria administrado por la Oficina de Servicios
Generales y estaria abierto permanentemente.

La propuesta no fue aprobada, enhorabuena. Sin embargo, evidencia la fuerza que tienen esas personas y grupos, pues se sienten con la potestad de pedir un lugar físico permanente para sus cultos dentro del espacio universitario, el cual -reitero- se supone que es laico, científico, autónomo y no debería tener relación alguna con la religiosidad (a menos que sea para estudiarla como fenómeno humano y social).

Pese a no tener un espacio oficial destinado a las prácticas religiosas en la UCR, de igual manera estas suceden. Así lo cuentan varias estudiantes: una dice que, en su escuela, hay "una señora que al medio día tiene charlas de oración, de leer la biblia, ahí en un espacio en la u" (Leonor). Y el detalle es que estas personas no se guardan sus prácticas y creencias para sí mismas, sino que las tratan de imponer a lxs demás. Lo grave del caso en el contexto universitario es que utilizan y se aprovechan de los medios/recursos institucionales -es decir, fondos públicos- no solo para realizar sus actividades, sino para divulgarlas e imponerlas.

Otra estudiante comenta que, en su escuela, a través de los correos electrónicos donde envían información de interés para la población estudiantil y egresada (por ejemplo, anuncios de trabajo y convocatorias a

\footnotetext{
9 Tomado del sitio web del VII Congreso Universitario, en el espacio donde se encuentran todas las ponencias realizadas (es decir, las propuestas presentadas para la universidad). Disponible en: <http://setimocongreso.ucr.ac.cr/sites/default/files/ponencias/VU-10.pdf>
} 
actividades universitarias), "han mandado correos invitando a las actividades estas evangélicas" (Ada). Según cuenta, se trata de lecturas bíblicas y conversación; quien está a cargo de ello es una profesora que cuenta su "historia de redención" a estudiantes que pasan por sus cursos: "yo era lesbiana, y gracias a Jesús o a la biblia o yo no sé que, me logré encontrar, gracias al amor infinito de Jesús" (Ada, estudiante). Dicha profesora es la responsable de cursos no solo en su escuela, sino para la universidad en general. De hecho, durante el diálogo con un grupo de estudiantes, cuando Ada hablaba sobre la susodicha historia, "que ella era lesbiana y que ella logró encontrar el camino y que las invitaba" a hacer lo mismo (ídem), Leonor -otra estudiante, incluso más joven- confirma el relato diciendo "no solo usted sabe esa historia digamos, que le contaron, a mí me lo dijo una chica que era lesbiana y que ella [la profesora] dijo 'yo era lo que usted era' ". Esto evidencia un profundo irrespeto hacia la vida de Ixs estudiantes por parte de la profesora, pues intenta llevarlas hacia 'el camino' que ella ha seguido, insistiendo en sus creencias religiosas.

Vemos en lo anterior un uso de su lugar de autoridad-poder para diseminar ideas ajenas a lo que corresponde en la universidad. Es decir, la labor docente es formar sujetxs para la vida profesional, personal, social y comunitaria, pero nunca adoctrinar en los principios religiosos en que él o ella cree. Porque -repito- la universidad es laica. La gravedad del asunto es que el caso de esa profesora no es aislado, es lo que sucede día a día en la universidad; y yo me atrevería a decir que mucho más de lo que sabemos o imaginamos. Por ejemplo, una tercera estudiante comparte otra experiencia con gran indignación: "en Educación más bien había cursos que iniciaban con una oración, en plena UCR digamos, es como un gueto del conservadurismo más puro y duro que hay" (Sofía). De nuevo, estamos ante el uso del espacio universitario y su lugar de poder (la figura docente) para imponer prácticas religiosas.

Esa realidad no se da sólo en los espacios de los cursos (en el ámbito curricular, si se quiere), sino que está en la estructura y la vida universitaria misma. Existen otras prácticas religiosas, católicas -mejor dicho-, que se dan en la universidad y -peor aún- están institucionalizadas en ella: los rezos del niño y el concurso de portales. Cada año, según la costumbre del catolicismo, en noviembre-diciembre se elaboran portales (pesebres, nacimientos) en las distintas oficinas de la universidad y se hace un concurso a nivel institucional (bueno, en la sede Rodrigo Facio) para premiar al mejor. El concurso es organizado ipor una vicerrectoría!, la Vicerrectoría de Acción Social (VAS); se realiza desde hace aproximadamente 20 años (sí, dos décadas) e, inclusive, actualmente tiene 
dos categorías para la premiación (Portal Tradicional y Portal Libre). Unido a ello, dado que se tienen los portales, la práctica católica es que para quitarlo, hay que hacer el rezo del niño; así se hace en la universidad. Cada oficina donde se elaboró un pesebre, organiza y ejecuta -en tiempo laboral, pagado con recursos públicos- dicha actividad religiosa. Respecto de todo esto, como bien lo dicen dos profesorxs: "eso está súper mal, súper mal" (Adriana) y "eso es violento [...] ¿por qué tienen que hacer eso?" (Mar).

Relato lo anterior puesto que, además del conocimiento que poseo de la universidad (por mi trayectoria en ella) y de lo que las personas comentaron en los diálogos, esa misma dinámica religiosa interna -que dificulta las labores académicas- se hizo presente durante el trabajo de campo: uno de los diálogos se vio interrumpido y fuertemente dificultado porque en una biblioteca de la universidad estaban realizando el rezo católico, incluso con música a alto volumen (y resalto: ¡en una biblioteca de la universidad!). Es decir, lo que surgió en los diálogos con las personas (porque de hecho el tema de la religiosidad estuvo presente en distintos grupos), lo constaté luego como experiencia y evidencia tangible en el transcurso de esta investigación. Pareciera que el dominio religioso dentro de la UCR no quiere dejar duda alguna de su existencia.

Y, resalto esta hegemonía religiosa porque sus enseñanzas están en la base -entre otras cosas- de las represiones y los mandatos en torno a la sexualidad, particularmente en el establecimiento de las normas de género patriarcales. No podemos desligar el machismo de la religión; incluso, si se quiere, este tipo de doctrinas son un fundamento importante para el primero.

\section{Reflexión final}

La naturalización de prácticas culturales pasa por la escuela (Louro, 2014), entendida como el campo de lo educativo formal. La universidad es parte de ese sistema pedagógico y pedagogizante, por tanto, contribuye en la creación y mantenimiento de esas prácticas. Esto es evidente en las vivencias que las personas comparten, a partir de sus experiencias dentro de la UCR. Las universidades deben trabajar para reducir al máximo la distancia creada por los dispositivos sociales. Lamentablemente, la UCR actúa como "una universidad que en lugar de estar incentivando el conocimiento, está incentivando una serie de prejuicios, mitos" (Diana, administrativa). Ello, pese a que el discurso que se asume es el de una 
universidad humanista. Cabe entonces pensar las preguntas que lanza Diana: "¿qué tanto de ese decir estamos haciendo? ¿por qué se siguen reproduciendo esos estigmas que en la sociedad existen, solo que a nivel micro?" Ello provoca "la enajenación de un sector poblacional que ha sido históricamente marginado y relegado [...] mientras se mantenga la distancia social habrá un mayor grado de rechazo" (Toro-Alfonso et al, 2008, p. 272). La consecuencia final de todo esto son las múltiples formas de discriminación y LGBTIfobia. 


\section{BIBLIOGRAFÍA}

Abelove, Samantha. (2015). "Coming Out of the Margins: LGBTI Activists in Costa Rica and Nicaragua". En: Scripps Senior Theses, Paper 524. Claremont, Estados Unidos. Disponible en: $<$ htt://scholarship.claremont.edu/scripps_theses/524>

Cornejo Espejo, Juan. (2012). "Componentes ideológicos de la homofobia". En: Límite, Revista de Filosofía y Psicología, Vol. 7 (Núm.26): pp.85-106. Disponible en: <http://www.redalyc.org/articulo.oa?id=83625847006>

García Ferrando, Urko Álex. (2015). "Teoría de los marcos de género". En: Pié, Asun \& Planella, Jordi (coords.). Políticas, prácticas y pedagogías traNs. Barcelona: Editorial UOC: pp.41-61.

Louro, Guacira Lopes. (2014). Gênero, sexualidade e educação: uma perspectiva pós-estruturalista. 16a edición. Petrópolis, RJ, Brasil: Vozes.

Margulis, Mario. (1997). "Cultura y discriminación social en la época de la globalización". En: Nueva Sociedad, (Núm.152): pp.37-52.

Meyer, Dagmar Estermann. (2013). "Gênero e educação: teoria e política". En: Louro, Guacira Lopes; Felipe, Jane; Goellner, Silvana Vilodre (orgs.). Corpo, gênero e sexualidade: um debate contemporâneo na educação. Novena edición. Petrópolis, RJ, Brasil: Vozes: pp. 11-29.

Molina Jiménez, Iván. (2015). Costarricense por dicha: identidad nacional y cambio cultural en Costa Rica durante los siglos XIX y XX. Primera edición, quinta reimpresión. San José: Editorial UCR.

Rosabal-Coto, Guillermo. (2016). Music learning in Costa Rica: A postcolonial institutional ethnography. Finlandia: Studia Musica 68 - The Sibelius Academy of the University of the Arts Helsinki.

Salas, José Manuel \& Campos, Álvaro. (2002). "Psicoerectus: los hombres y su vivencia cotidiana de la sexualidad". En: José Manuel Salas y Álvaro Campos (comps.). Masculinidades en Centro América. Primera edición. San José, Costa Rica: Lara Segura Editores: pp. 201-217. 
Toro-Alfonso, José ; Borrero Bracero, Néstor ; Nieves Lugo, Karen. (2008). "De la exclusión al estilo universitario: la homofobia en la Universidad de Puerto Rico". En: Revista Análisis, Vol.IX (Núm. 1): pp. 269-295. 